\title{
Predictors of osteoporosis preventive behaviors among adolescent: a cross-sectional study
}

Predyktory zachowań zapobiegających osteoporozie wśród młodzieży: badanie przekrojowe

\author{
${ }^{1}$ Faezeh Rastgoo, ${ }^{1}$ Parvaneh Vasli, ${ }^{2}$ Camelia Rohani, ${ }^{3}$ Aminreza Amini
}

${ }^{1}$ Department of Community Health Nursing, School of Nursing and Midwifery, Shahid Beheshti University of Medical Sciences, Tehran, Iran

${ }^{2}$ Affiliated Researcher, Department of Health Care Sciences, Palliative Research Center, Ersta Sköndal Bräcke University College, Campus Ersta, Stockholm-Sweden

${ }^{3}$ Department of Biostatistics, Faculty of Paramedical, shahid Beheshti University of Medical Sciences, Tehran, Iran

\begin{abstract}
Background: Osteoporosis is a preventable progressive metabolic disease. Girls have an increased risk of occurrence of osteoporosis in their old age. The BASNEF model can be employed to change behaviors related to health.

Aim of the study: The BASNEF model was employed to determine the predictors of osteoporosis preventive behaviors among adolescent girls.

Material and methods: This cross-sectional study was carried out on 209 adolescent girls selected from high schools in the Quchan County in 2016 using path analysis by stratified sampling. The data was collected through a demographic questionnaire and a 52 -item researcher-made questionnaire, based on the BASNEF model constructs. The data was analyzed using Shapiro-Wilk test, bootstrapping, and path analysis.

Results: The average age of the students was $16.10 \pm 0.59$. The results of path analysis showed that Model 1 matched the BASNEF model relationships completely; however, it could not predict osteoporosis preventive behaviors. The constructs of Model 2 (modified) was able to predict $50 \%$ of variances in osteoporosis preventive behaviors. There were positive and direct relationships between the following pairs of constructs: knowledge and attitudes $(B=0.23, p<0.001)$; attitudes and the intention of osteoporosis preventive behaviors $(B=0.37, p<0.001)$; subjective norms and the intention of osteoporosis preventive behaviors $(B=0.53, p<0.001)$; behavioral intention and osteoporosis preventive behaviors $(B=0.36, p<0.001)$; subjective norms and osteoporosis preventive behaviors $(B=0.33, p<0.001)$, and enabling factors and osteoporosis preventive behaviors $(B=0.29, p<0.001)$.
\end{abstract}

Conclusions: The community health nurse can use the constructs of the BASNEF model to change the osteoporosis preventive behaviors like knowledge, attitudes subjective norms and enabling factors.

Key words:

adolescent, community health nurses, osteoporosis, causality.

\section{Introduction}

Osteoporosis is a progressive metabolic disease reducing bone mass and strength which can cause death and adversely affect the quality of life [1]. Osteoporosis is regarded as a serious problem in developed and developing countries [2]. This disease affect $30 \%$ of postmenopausal in the USA and the proportion rises to $70 \%$ in women over the age of 80 years [3]. According to the reports provided by rheumatology research centers in Iran, six million Iranians suffer from osteoporosis [4].
Women are four times more likely than men to be affected by osteoporosis, which is known as a major threat to the public health, especially among women [5]. Compared with Western countries, Asians have a lower BMI (body mass index); thus, they are more prone to osteoporosis [6]. Bone mass and bone density usually reach a peak during the childhood and adolescence of both genders until the age of thirty [7]. Since bone density decreases as a person ages, obtaining the maximum bone mass in the first three decades of life and maintaining it during the middle age can significantly decrease the chance 
of osteoporosis [8]. On the other hand, adolescent health impairments may have negative long-term outcome for the health of the entire community [9]. To prevent osteoporosis, girls are important, since they have an increased risk of occurrence of osteoporosis in their old age [10].

Researchers have benefited from health educational models to identify the factors affecting behaviors. Such models have been devised to help diagnose and perceive factors affecting behaviors and determine what these factors do [11]. Among the models presented, BASNEF model is one of the most comprehensive health models used to examine the behaviors and to identify the behavior and development of new behaviors in community [12]. The BASNEF model can be employed to change behaviors for health promotion by improving individual skills and creating a kind of support and empowerment [13]. The BASNEF model is made up of the PRECEDE model and the behavioral intention model. It includes certain constructs such as attitudes, subjective norms, enabling factors, behavioral intention, and behavior. According to the BASNEF model, people evaluate a new behavior when they believe it is useful. Then, they develop attitudes. In parallel with this trend, key individuals can have facilitating or inhibiting roles in decisions made by people. A combination of attitudes and subjective norms result in a certain behavioral intention to develop a new behavior. On the other hand, a behavior can be under the influence of other factors such as skills, money, and expenditures, known as enabling factors [13, 14]. In Iran's National Health Network System, this model has been introduced as the official behavioral change and planning model [15]. Due to its various applications in health, this model has been taken into special account by international health organizations such as UNICEF and WHO. The results of using this model in Asian and African countries have shown significant successes [16].

A number of studies have also used the BASNEF model for assessing factors influencing health behaviors and the effect of education on health behaviors among adolescent girls, young people and women. Rostami Moez et al. used the BASNEF model to examine factors affecting the regular physical activity of high school girls in Hamadan city, Iran [15]. However, they only studied the physical activity of girls and did not address other aspects of prevention behaviors of osteoporosis. Another study was conducted to assess the predictors of osteoporosis prevention in women. These studies have two differences with the current study as follow: their samples were only women, also the health belief model was used to assess the predictors of health behaviors [17]. In another study, the BASNEF model was used to examine factors related to only physical activity in medical students in both male and female students [18]. Generally, the review of literature showed no studies on the predictors of osteoporosis prevention behaviors based on the BESNEF model.

Nevertheless, the researchers could not find any studies on the predictors of osteoporosis preventive behaviors based on the BASNEF model among adolescent girls. Given the significance of osteoporosis preventive behaviors among adolescent girls, this study was conducted on adolescent girls of Quchan
County, Iran, to analyze the predictors of preventive behaviors based on the BASNEF model.

\section{Material and methods}

This cross-sectional study was carried out in 2016 by path analysis. For several reasons, path analysis is preferred over regression models. Basically, several theoretical models are hypothesized in the path analysis and are examined. A regression model incorporates only variables, where a single variable is predicted and explained by one or more independent variables. Due to path analysis flexibility, it is possible to investigate several independent variables and several dependent variables. Path analysis also enable us to measure the direct and indirect effect that one variable has upon another [19]. Path analysis as a series of regression equations also can present the causal processes under study in the form of an image scheme and, by symbolizing one-way flashes, shows how variables affect each other [20].

The participants of the study were the adolescent girls selected from the high schools. According to literature, for conducting path analysis, at least 200 individuals are required [21]. Considering $10 \%$ sample attrition, the initial number of participants was reported as 220 people. During the data analysis, 11 questionnaires were excluded due to incompleteness, and therefore, the data analysis was performed with 209 participants.

The stratified sampling method was used to select the participants. For this purpose, Quchan County was first divided into four geographical regions, from each of which one female high school was selected at random. In the next step, a list of students was provided at each designated high school. Then a number was allocated to every student meeting the inclusion criteria. In total, 52 adolescent girls were selected through simple random sampling from each of the three designated high schools; however, 53 students were selected from one high school. The inclusion criteria: studying in the first high school grade and no history of diseases such as musculoskeletal disorders, fractures, diabetes, and asthma reported by the participants themselves.

The dependent variable was the osteoporosis preventive behavior; however, the independent variables were BASNEF constructs such as knowledge (knowledge about prevention of osteoporosis), attitudes (attitude toward prevention of osteoporosis), subjective norms (beliefs and expectations of families, friends, acquaintances regarding compliance with osteoporosis preventive principles), behavioral intention (willingness of participants to show osteoporosis preventive behaviors), enabling factors (A: individual-familial resources; B) external and environmental enabling resources) and behavior (osteoporosis preventive behaviors include physical activities, calcium-rich diets, use of sunlight, and consumption of vitamin D pills).

The data collection instruments were a demographic questionnaire and a researcher-made questionnaire based on the BASNEF model. Given the fact that knowledge leads to commitment to health-related activities [22], and increasing knowl- 
edge can change behaviors [23], knowledge was regarded as a construct predicting osteoporosis preventive behaviors in the modified model. The demographic questionnaire included 11 items (age, major, number of family members, birth rank, having living parents, educational attainments of parents, occupations of parents, suffering from a special disease, and a history of osteoporosis in family).

The researcher-made questionnaire was based on the latest available references and the BASNEF model [2, 4, 24]. It consisted of 6 sections including knowledge, attitude, subjective norms, enabling factors, behavioral intention, and intention with 52 items in total. There were 15 items on knowledge with responses like Correct, I don't know, and False with three scores, two scores, and one score, respectively. There were $6,7,8$, and 9 items on attitude, subjective norms, intention of osteoporosis preventive behaviors, and enabling factors, respectively. These items were ranked on a five-point Likert scale, ranging from / totally agree to I totally disagree, scored from one to five with respect to being direct or inversed. Finally, osteoporosis preventive behaviors were evaluated by using 7 expressions on a five-point Likert scale, ranging from Always to Never, scored from five to one.

The content validity analysis of the instrument was conducted to determine the scientific validity of the BASNEF-based questionnaire. For this purpose, the questionnaire was codified by using appropriate references and books. Then an expert panel of ten nursing faculty members was surveyed in order to determine the qualitative content validity and CVR (content validity ratio). According to Lawshe's table with such an expert panel, 0.62 is considered an acceptable value of CVR. Moreover, every item received a score above 0.8 ; therefore, no questions were left out. However, some of the items were revised with respect to qualitative validity and formal validity. Furthermore, the average CVI (content validity index) was acceptable in relevancy. Different values of CVI were reported 94.6, 88.5, 91.9, 100, 92.7, and 95.4 obtained for knowledge, attitude, subjective norms, behavioral intention, enabling factors, and osteoporosis preventive behaviors, respectively.

The reliability of the BASNEF-based questionnaire was analyzed by employing internal consistency, for which Cronbach's $\alpha$ was calculated, and stability, for which the test-retest method was used with ICC (intra-class correlation). In other words, questionnaires were filled out by 30 students, who were excluded from the study later, at two points with a 14-day gap. The values of ICC were obtained 0.62, 0.81, 0.89, 0.90, 0.87, and 0.91 for knowledge, attitude, subjective norms, behavioral intention, enabling factors, and osteoporosis preventive behaviors, respectively. Moreover, Cronbach's $\alpha$ was reported 0.71 , $0.73,0.76,0.78,0.82$, and 0.76 for knowledge, attitude, subjective norms, behavioral intention, enabling factors, and osteoporosis preventive behaviors, respectively.

With the cooperation of school officials, questionnaires were distributed among female students who were willing to participate in the study between class hours. The students were asked to fill out the questionnaires and return them. The researcher was present in classes where questionnaires were being filled out.
Data were collected after an authorization was issued by the Ethical Committee of Shahid Beheshti University of Medical Sciences (IR.SBMU.PHNM.1395) in addition to a written consent issued by the Education Department of Quchan County and the consent of school officials. The participants were informed of the research goals and methodology, confidentiality of information, voluntary participation, and free dismissal. They participated in this study at free will. The written consent form was filled out by participants. The questionnaires were filled out without mentioning any names and only with codes.

Descriptive indicators such as percentage, mean and standard deviation were used to analyze the research data. The Shapiro-Wilk test was conducted to evaluate the normality of variables with the help of kurtosis and skewness. Before the data analysis, the research data were checked to see whether they were outliers. The path analysis technique was employed in AMOS 22 to analyze the determinants of osteoporosis preventive behaviors based on the BASNEF model. It should be noted that bootstrapping was used to analyze the indirect effects including significance and confidence intervals. The significance level was considered 0.05 .

The ratio of $\chi^{2}$ to degree of freedom $\left(\chi^{2} / d f\right)$, goodness of fit index (GFI), comparative fit index (CFI), and root mean square error of approximation (RMSEA) were employed to analyze the goodness of fit in path analysis. According to different references, the fitness of a model can be accepted if the values of $\chi^{2} / \mathrm{df}$, RMSEA, GFI, and CFI are smaller than 3, smaller than 0.05, larger than or equal to 0.95 , and larger than or equal to 0.97 . Other indices were also used in this study such as normed fit index (NFI), non-normed fit index (NNFI), and adjusted goodness of fit index (AFGl), which are all acceptable if above $0.9[25,26]$.

\section{Results}

There were 209 students participating in this study (60 in mathematics; 58 in empirical sciences; and 111 in human sciences). The average age of the students was $16.10 \pm 0.59$. Other demographic pieces of information can be seen in Table I. Descriptive indicators including mean and standard deviation were as follows: knowledge (19 \pm 5$)$; attitudes (24.7 \pm 3.9$)$; subjective norms (24.2 \pm 4.3$)$; behavioral intentions (31.6 \pm 5.3$)$; enabling factors $(31 \pm 5.6)$; osteoporosis preventive behaviors $(23 \pm 5.1)$.

At first, path analysis was employed to analyze the factors affecting osteoporosis preventive behaviors based on the BASNEF model. Therefore, Model 1 was extracted. However, some goodness of fit indices were not confirmed $\left(\chi^{2} / \mathrm{df}=16.116\right.$, $\mathrm{GFI}=0.839, \mathrm{AGFI}=0.598, \mathrm{CFI}=0.631, \mathrm{NFI}=0.622$, $\mathrm{NNFI}=0.386$, RMSEA $=0.270)$. In the next step, the goodness of fit indices of Model 2, which was the modified version of Model 1, were confirmed in order to obtain a better goodness of fit index and determine direct and indirect effects of constructs on each other and on the dependent variable, i.e. osteoporosis preventive behaviors. The values of fitness indices of model 2 are as follows: $\chi^{2} / \mathrm{df}=1.317, \mathrm{GFI}=0.984, \mathrm{AGFI}=0.957$, $\mathrm{CFI}=0.990, \mathrm{NFI}=0.962, \mathrm{NNFI}=0.982$, and $\mathrm{RMSEA}=0.039$. The models 1 and 2 shown in Figs. 1 and 2, respectively. 
Table I. Demographic information of participants

\begin{tabular}{|c|c|}
\hline Demographic Variables & $n(\%)$ \\
\hline \multicolumn{2}{|l|}{ The number of family members } \\
\hline 3 or less & $10(4.8)$ \\
\hline 4 & $53(25.4)$ \\
\hline 5 & $86(41.1)$ \\
\hline 6 people or more & $60(28.7)$ \\
\hline \multicolumn{2}{|l|}{ Birth rank } \\
\hline The first child & $87(41.8)$ \\
\hline The second child & $54(25.8)$ \\
\hline The third child & $26(12.4)$ \\
\hline The fourth or later child & $2(20.1)$ \\
\hline \multicolumn{2}{|l|}{ Having living parents } \\
\hline Yes & $198(94.7)$ \\
\hline No & $11(5.3)$ \\
\hline \multicolumn{2}{|l|}{ Parental education } \\
\hline Illiterate & $8(3.8)$ \\
\hline Elementary school and junior high school & $95(45.4)$ \\
\hline Senior high school an diploma & $89(42.6)$ \\
\hline Academic & $17(8.1)$ \\
\hline \multicolumn{2}{|l|}{ Maternal education } \\
\hline Illiterate & $16(7.7)$ \\
\hline Elementary school and junior high school & $104(49.8)$ \\
\hline High school and diploma & $80(38.3)$ \\
\hline Academic & $9(4.3)$ \\
\hline
\end{tabular}

\begin{tabular}{|c|c|}
\hline Demographic Variables & $n(\%)$ \\
\hline \multicolumn{2}{|l|}{ Paternal occupation } \\
\hline Clerk & $40(19.1)$ \\
\hline Self-employed & $129(61.7)$ \\
\hline Unemployed & $9(4.3)$ \\
\hline Retired & $28(13.4)$ \\
\hline \multicolumn{2}{|l|}{ Maternal occupation } \\
\hline Clerk & $13(6.2)$ \\
\hline Self-employed & $9(4.3)$ \\
\hline Retired & $3(1.4)$ \\
\hline Housewife & $184(88)$ \\
\hline \multicolumn{2}{|l|}{ A history of osteoporosis in family } \\
\hline Yes & $25(12)$ \\
\hline No & $101(48.3)$ \\
\hline I don't know & $83(39.7)$ \\
\hline \multicolumn{2}{|l|}{ Information source } \\
\hline Public media such as TV and Radio & $44(21.1)$ \\
\hline Educational books and brochures & $7(3.3)$ \\
\hline The Internet & 7 (3.3) \\
\hline School health instructor & $18(8.6)$ \\
\hline Doctors and other healthcare staff & $4(2.5)$ \\
\hline Others & $14(6.7)$ \\
\hline
\end{tabular}

The first part of Table II shows the raw and standardized coefficients of direct interplays between constructs and paths of Model 2 (modified). According to the Table II and Figure 2, if knowledge increases by one unit, attitude will increase by 0.23 ( $p<0.001)$. If attitude increases by one unit, behavioral intention increases by $0.37(p<0.001)$. If subjective norms increases by one unit, behavioral intentions will increase by 0.53 $(p<0.001)$. If behavioral intentions increase by one unit, osteoporosis preventive behaviors will increase by $0.36(p<0.001)$. If enabling factors increase by one unit, osteoporosis preventive behaviors will increase by $0.29(p<0.001)$. Finally, if subjective norms increase by one unit, osteoporosis preventive behaviors will increase by $0.33(p<0.001)$. According to the standard coefficient of $\beta$, it can be concluded that the greatest direct effect was made by subjective norms on the intention of osteoporosis preventive behaviors $(p<0.001)$. Among the constructs having direct effects on osteoporosis preventive behaviors, behavioral intentions had the greatest effect (0.37).

According to the second part of Table II which also shows raw and standardized coefficients of indirect interplays between constructs in Model 2 (modified), it can be concluded that if knowledge increases by one unit, attitudes will increase by 0.23 , and if attitudes change by one unit, then behavioral intention will increase by 0.37 ; in addition, if behavioral inten- 


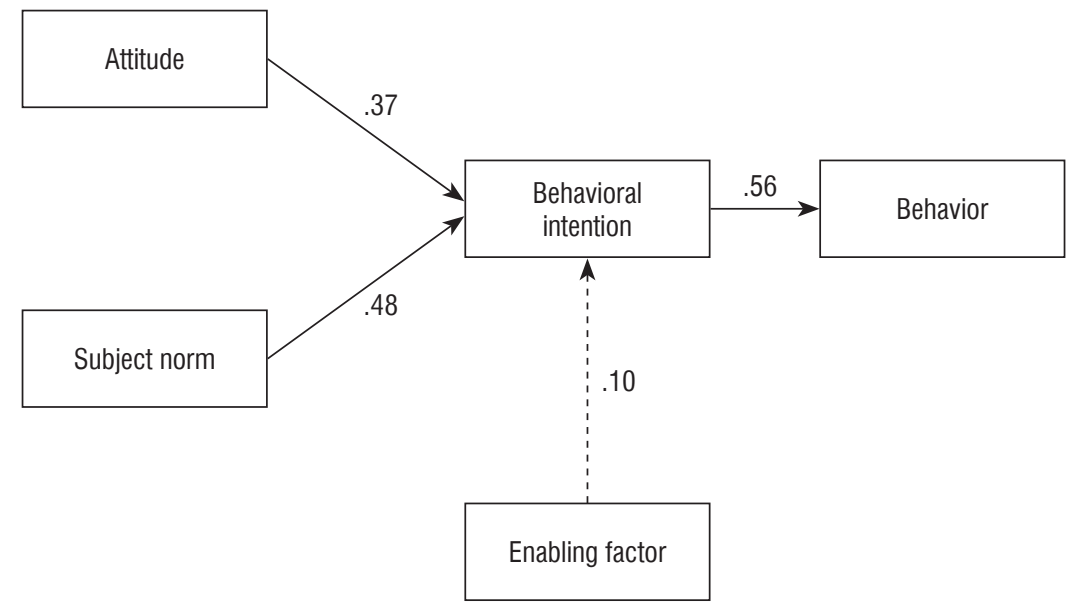

Figure 1. Path analysis based on BASNEF constructs (Model 1)

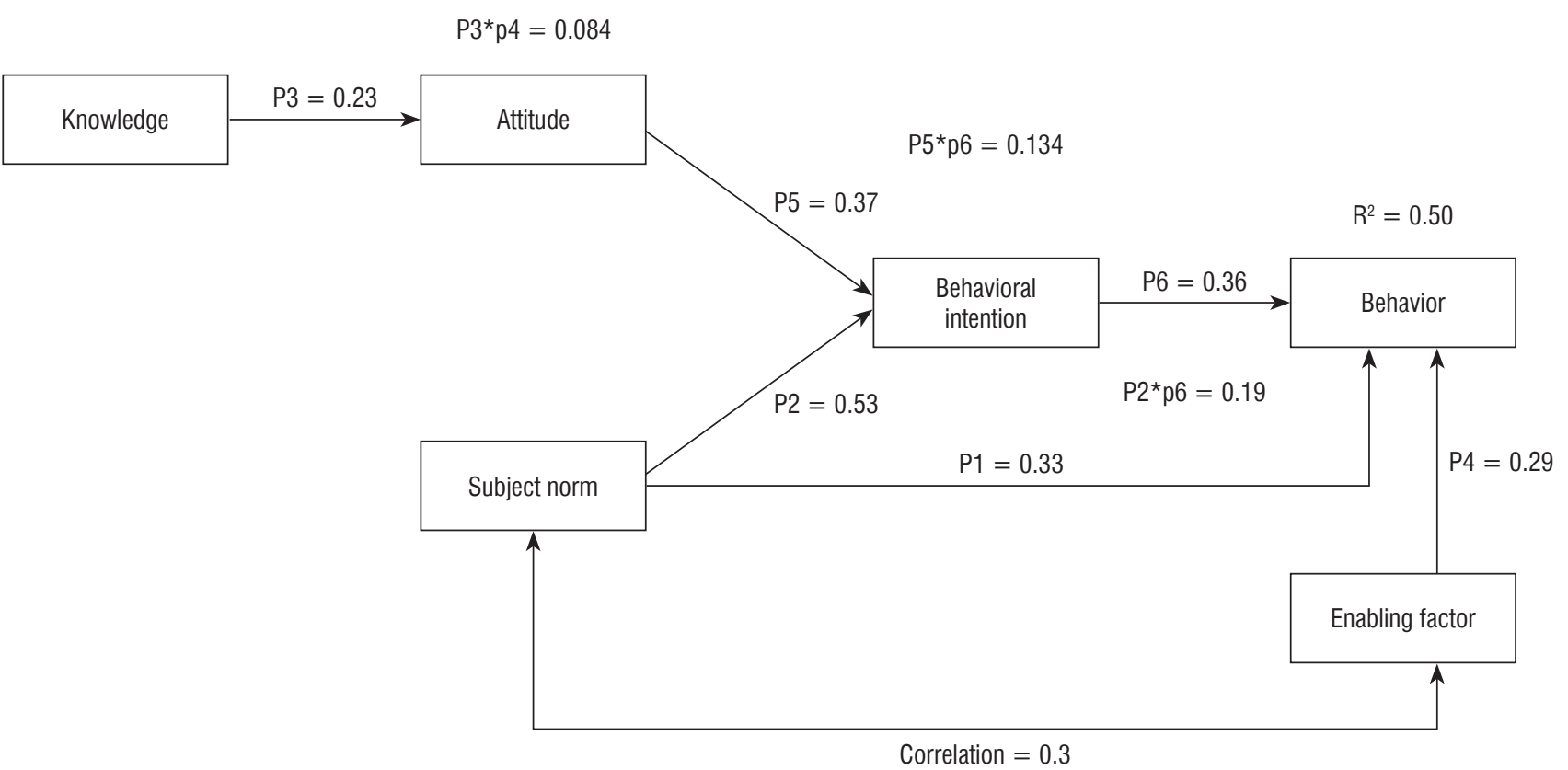

Figure 2. Modified path analysis based on BASNEF constructs (Model 2)

tion increase one unit, then osteoporosis preventive behaviors will increase by 0.36 . The results also show that the average score of behavioral intention of people with higher knowledge scores will indirectly increase by 0.08 units with an intermediary role of attitude $(p=0.001)$. Furthermore, the average score of osteoporosis preventive behaviors will increase by 0.03 units if attitude and behavioral intention increase indirectly with tan intermediary role of attitudes and behavioral intentions $(p<0.001)$. Moreover, if subjective norms increase by one unit, behavioral intentions will increase by 0.53 units. If the score of behavioral intention increases by one unit, the score of preventive behaviors with increase by 0.36 , something which shows that the average score of osteoporosis preventive behaviors increases nearly 0.19 units with the intermediary role of behavioral intentions for the people whose average score of behavioral intention is one unit higher $(p=0.002)$. Moreover, if attitudes increase by one unit, behavioral intention will increase by 0.37 , and if behavioral intention increases by one score, then osteoporosis preventive behaviors will increase by 0.36 $(p=0.001)$. The findings indicated that the average score of osteoporosis preventive behaviors would increase by 0.13 for people whose attitude scores were one unit higher $(p=0.001)$. 
Table II. Raw and standardized coefficients of direct interplays between constructs and paths of Model 2 (modified)

\begin{tabular}{|c|c|c|c|c|c|c|c|}
\hline & Path & $\mathrm{B}$ & SE & Beta & $t$ & $P$-value & $\begin{array}{l}P \text {-value for whole } \\
\text { of model }\end{array}$ \\
\hline \multicolumn{7}{|l|}{ Direct effect } & \multirow{12}{*}{$\begin{array}{l}\chi^{2}=10.5 \\
p=0.22\end{array}$} \\
\hline Energy 2000-2600 kcal & P3 & 0.23 & 0.52 & 0.29 & 4.38 & $<0.001$ & \\
\hline Carbohydrates $45-50 \%$ & P2 & 0.53 & 0.071 & 0.43 & 7.37 & $<0.001$ & \\
\hline Simple sugars $<10 \%$ & P5 & 0.37 & 0.07 & 0.27 & 4.68 & $<0.001$ & \\
\hline Dietary fibre $20-40 \mathrm{~g}$ & P6 & 0.36 & 0.05 & 0.37 & 6.86 & $<0.001$ & \\
\hline Protein $15-20 \%$ & $\mathrm{P} 4$ & 0.29 & 0.05 & 0.30 & 5.83 & $<0.001$ & \\
\hline Fats $30-35 \%$ & $\mathrm{P} 1$ & 0.33 & 0.06 & 0.28 & 4.95 & $<0.001$ & \\
\hline \multicolumn{7}{|l|}{ Indirect effect } & \\
\hline Knowledge to behavioral intention & $\mathrm{P} 3 * \mathrm{P} 5$ & 0.08 & 0.02 & 0.08 & & 0.001 & \\
\hline Knowledge to behavior & $\mathrm{P} 3{ }^{\star} \mathrm{P} 5{ }^{\star} \mathrm{P} 6$ & 0.03 & 0.01 & 0.03 & & $<0.001$ & \\
\hline Subjective norms to behavior & $\mathrm{P} 2 * \mathrm{P} 6$ & 0.18 & 0.04 & 0.16 & & 0.002 & \\
\hline Attitudes to behavior & $\mathrm{P} 5{ }^{\star} \mathrm{P} 6$ & 0.13 & 0.04 & 0.104 & & 0.001 & \\
\hline
\end{tabular}

In a general conclusion based on Model 2 (Fig. 2) and Table II, subjective norms had the greatest effect on osteoporosis preventive behaviors indirectly with the intermediary of behavioral intentions with a standard $\beta$ of 0.16 . Finally, the research findings indicated that the BASNEF constructs could predict $50 \%$ of the variance of osteoporosis preventive behaviors.

\section{Discussion}

For data analysis, the effects of important constructs on osteoporosis preventive behaviors were first drawn to match the BASNEF model (Model 1). However, the results of fitness did not confirm the sufficiency of the model. In fact, constructs could not affect each other and osteoporosis preventive behaviors in compliance with the BASNEF model (Model 1) in the statistical population. In the next step, knowledge was entered the model, and the interplay of constructs was changed in order to confirm Model 2 (modified) which was different from the BASNEF model in some aspects.

According to the BASNEF model, it is obvious that a combination of attitudes and subjective norms would form the behavioral intention to develop a new behavior. Moreover, enabling factors affect the path between a behavioral intention and a behavior. However, some of such paths were not confirmed in Model 2 (Fig. 1). In addition, knowledge had a direct effect on attitudes. Furthermore, subjective norms affected the behavioral intentions along with attitudes. Such norms also affected preventive behaviors. Enabling factors had a direct effect on osteoporosis preventive behaviors, too. According to the re- search findings, knowledge had a direct effect on attitude. It had an indirect and poor effect on osteoporosis preventive behaviors through the intermediary roles of attitude and behavioral intention. A brief review of previous studies indicated that knowledge affected attitude. Heshmati et al. carried out a study to analyze the factors related to the educational behaviors of healthcare workers on oriental sores based on the BASNEF model. The results showed that there was a direct relationship between knowledge and attitude [27]. The another study showed a significant relationship between knowledge and the intake of calcium with physical activities [28]. The relationship between knowledge and health behaviors including the osteoporosis preventive behavior, can be attributed to the effect of knowledge on the change in individuals' attitudes [29].

According to the other findings, attitudes had positive effects on osteoporosis preventive behavioral intention and had indirect effects on preventive behaviors through the intermediary role of the same construct. Rostami Moez et al. used the BASNEF model to analyze the factors affecting physical activities of high school girls. They showed that there was a significant relationship between attitude and behavioral intention [15]. Therefore, it appears necessary to make changes in knowledge, attitude, and culture to improve health-promoting behaviors in society [30].

In this study, the greatest direct interplay of constructs came from the rather significant effectiveness of subjective norms on osteoporosis preventive behaviors. Tabatabaei et al. indicated that subjective norms had direct effects on behavioral intention. They also showed that subjective norms had the greatest effect 
on osteoporosis preventive behaviors through the intermediary role of intention [31]. Rostami Moez et al. indicated that subjective norms affected physical activities through behavioral intention [15]. Subjective norms such as friends and the family can affect the behavioral intention of adolescents. As a result, they encourage them to engage in positive behaviors, that increases the likelihood of occurrence of behaviors.

In this study, the intention of osteoporosis preventive behaviors was regarded as the most powerful construct affecting osteoporosis preventive behaviors. Tabatabaei et al. indicated that behavioral intention had direct effects on physical activity [31]. Moreover, Asadi and Salehi indicated that there was a correlation between behavioral intention and behavior and that behavioral intention affected behavior [28]. Given these findings, strengthening behavioral intentions can increase the likelihood of osteoporosis preventive behaviors.

According to the research findings, enabling factors had direct effects on osteoporosis preventive behaviors. Other studies indicated that enabling factors were good predictors of osteoporosis preventive behaviors. For instance, Darsareh et al. found out that increasing the scores of enabling factors would increase physical activities [32]. Naseri Pour Takallo et al. indicated that the most powerful predictor was enabling factors among the BASNEF constructs. It had the strongest relationship with oral health behaviors [33]. In fact, it can be concluded that enabling factors had a major role in creating preventive behaviors.

Finally, research findings showed that the constructs of Model 2 of the BASNEF model (modified) could predict $50 \%$ of variance in osteoporosis preventive behaviors. Kabodi et al. showed that BASNEF constructs could predict $24 \%$ of variance in self-treatment behaviors [34]. Quji et al. indicated that knowledge, attitude, and subjective norms were the predictors of physical activities by explaining $21 \%$ of the variance in behavioral intention. Therefore, behavioral intention was the best predictor of postnatal physical activities [35]. In total, it can be stated that the BASNEF model could predict $50 \%$ of variance in osteoporosis preventive behaviors. In other words, the constructs of this model had significant roles in developing osteoporosis preventive behaviors among adolescent girls. There-

\section{References}

1. Marcus R, Dempster DW, Cauley JA, Feldman D. Osteoporosis. Academic Press 2013.

2. Kahnamouei-aghdam F, Amani F, Farzaneh E, Vejdani M. Female students' awareness of osteoporosis in Ardabil city. International Journal Of Community Medicine and Public Health 2017; 2: 323-327.

3. Meeta. Post-Menopausal Osteoporosis: JAYPEE 2013.

4. Moghimi J, Safaei Z, Behnam B, Ghorbani R. Knowledge Towards Prevention of Osteoporosis in Adolescent Girls: Effect of Educational Program. Middle East Journal of Rehabilitation and Health 2017; 4: e41622.

5. Sharifi N, Majlessi F. Self-empowerment of female students in prevention of osteoporosis. Global Journal of Health Science 2016; 9: 7. fore, changing any of these constructs, especially subjective norms related to the effects of families, relatives, and acquaintances, would facilitate osteoporosis preventive behaviors.

There were certain research limitations include the effect of mental status and fatigue of participants on responses given to the items in addition to the evaluation of osteoporosis preventive behaviors on self-report scales.

\section{Conclusions}

According to the research findings, the constructs of a BASNEF-modified model could relatively predict osteoporosis preventive behaviors. For this purpose, it is suggested that continuous and regular educational plans be devised by the public media and schools to change attitudes and knowledge of society, families, and female students. On the other hand, given the fact that subjective norms had the greatest indirect effect on osteoporosis preventive behaviors, certain public classes can be held for female students to improve the effectiveness of peers. Moreover, certain classes can be held for the families of adolescent girls to improve osteoporosis preventive behaviors.

The research findings also showed that osteoporosis preventive behaviors could be directly improved by providing individual-familial enabling factors and external enabling factors such as the access to the Internet and the ability to use it. Other important factors are the financial capability of a family to provide calcium-rich foods, the use of gyms, and the availability of appropriate sports equipment at homes and schools with the help of health instructors.

Given the fact that the results of this study showed that the modified BASNEF model could partly predict osteoporosis preventive behaviors of adolescent girls, it is recommended that community health nurses use this model to empower and improve the health of adolescent girls to prevent osteoporosis.

The authors also suggest that other studies be conducted by using this model to predict factors affecting other healthrelated behaviors of adolescent girls such as physical activities and eating habits so that more effective interventions could be designed and implemented.
6. Ahmad MS, Mohamed IN, Mokhtar SA, Shuid AN. Review of the risk factor of osteoporosis in the Malaysian population. RUMes 2014; 3: 77-82.

7. Al Seraty WHH, Ali W. The impacts of health belief model based intervention for osteoporosis prevention among female students in $\mathrm{Al}$ Dawadmi Applied Medical Science, Shaqraa University, Saudi Arabia. Journal of Biology, Agriculture and Healthcare 2014; 4: 125-131.

8. Malak MZ, Toama ZT. The effect of osteoporosis health education program based on health belief model on knowledge and health beliefs towards osteoporosis among Jordanian female teachers. European Scientific Journal 2015; 11: 385-395.

9. Rashidi Fakari F, Simbar M, Ghasemi V, Saei Gharenaz M. Inhibitors and Facilitators of Unwanted Adolescent Pregnancy in Iran and the World: A Review. Evidence Based Care 2017; 7: 59-70. 
10. Darabi L, Amin Shokravi F, Ghaffari M. Assessment of designed pamphlet of osteoporosis knowledge of girl students. HEHP 2015; 3: 27-36.

11. Brannon L, Feist J, Updegraff JA. Health psychology: An introduction to behavior and health: Cengage Learning 2013.

12. Masoumi SZ, Kazemi F, Ahmadi S. Investigating the Effects of Instructing Mothers Using BASNEF Model on Continuing Exclusive Breastfeeding of Late-Preterm Infants. J Compr Ped 2017; 8: e59243.

13. Ahmadi S, Kazemi F, Masoumi SZ, et al. Intervention based on BASNEF model increases exclusive breastfeeding in preterm infants in Iran: a randomized controlled trial. Int Breastfeed J 2016; 11: 30.

14. Zendehtalab H, Vaghei S, Emamimoghadam Z. Effect of intervention based on BASNEF model on quality of life in patients with type 2 diabetes. Evidence Based Care 2013; 3: 7-16.

15. Rostami Moez M, Hazavehei SM, Moeini B, Karimi Shahanjarini A, Roshanaei $G$. Effectiveness of educational program based on BASNEF model for using safe contraception methods. J Research Health 2014; 4: 705-713.

16. Saffari H, Shojaeizadeh D, Ghofranipour F, et al. Health education \& Promotion Theories, Models \& Methods. Sobhan, Tehran 2012.

17. Zareban I, Tavallai M, Shahrakipour M, et al. Prediction of osteoporosis preventive behaviors through the use of the Health Belief Model (HBM). HEHP 2016; 4: 55-62.

18. Alizadeh $\mathrm{S}$, Jadgal KM, Age Atabay Re, et al. Factors related to physical activity based on "BASENF Model" in Kerman university of medical sciences students. JHPM 2017; 6: 23-29.

19. Olobatuyi ME. A user's guide to path analysis: University Press of America; 2006

20. Garson GD. Path analysis: Statistical Associates Publishing Asheboro, NC; 2013.

21. Hoe SL. Issues and procedures in adopting structural equation modeling technique. JAQM 2008; 3: 76-83.

22. Kaur S. Osteoporosis in Indians: role of nurses in prevention. Maturitas 2009; 63: S95.

23. World Health Organization. Regional Office for the Eastern Mediterranean. Health education: theoretical concepts, effective strategies and core competencies: a foundation document to guide capacity development of health educators 2012. Available from: http://www. who.int/iris/handle/10665/119953.
24. Nguyen NV, Dinh TA, Ngo QV, Tran VD, Breitkopf CR. Awareness and knowledge of osteoporosis in Vietnamese women. Asia Pac J Public Health 2015; 27:NP95-NP105.

25. Hu Lt, Bentler PM. Cutoff criteria for fit indexes in covariance structure analysis: Conventional criteria versus new alternatives. Struct Equ Modeling 1999; 6: 1-55.

26. Byrne BM. Structural Equation Modeling with AMOS: Basic Concepts, Applications, and Programming: Routledge 2016.

27. Heshmati H, Rahaei Z, Hazavehei S, et al. Related factors to educational behaviors of health volunteers about cutaneous leishmaniasis on the basis of BASNEF model in Yazd. J Health 2010; 1: 48-56.

28. Asadi Shavaki M, Salehi L. Knowledge, belief and practice amongst elderly about osteoporosis based on the health belief model. Sci J Hamadan Nurs Midwifery Fac 2016; 24: 86-97.

29. Kassahun CW, Mekonen AG. Knowledge, attitude, practices and their associated factors towards diabetes mellitus among non diabetes community members of Bale Zone administrative towns, South East Ethiopia. A cross-sectional study. PloS One 2017; 12: e0170040.

30. Khodaveisi M, Omidi A, Farokhi S, Soltanian A. Dietary behavior status and its predictors based on the penders health promotion model constructs among overweight women referred to Fatemieh hospital clinics in Hamedan, 2014. JNE 2016; 5: 31-39.

31. Tabatabaei SVA, Ardabili HE, Haghdoost AA, et al. Factors affecting physical activity behavior among women in kerman based on the Theory of Planned Behavior (TPB). Iran Red Crescent Med J 2017; 19: e14057.

32. Darsareh F, Aghamolaei T, Ghanbarnejad A. Prediction of Physical Activity based on BASNEF Model Constructs among female teachers in Schools of Bandar Abbas. JPM 2015; 2: 1-9.

33. Naseri Pour Takallo Z, Ahmadi Tabatabaei SV, Zolala F, Mohseni M. Effective Factors on Oral Health-Promoting Behaviors among Elementary School Students Based on BASNEF Model. HBRJ 2016; 2: 259-273.

34. Kabodi S, Hazavehei MM, Rahimi M, Roshanaei G. Application of BASNEF model in analyzing self-treatment behavior among type 2 diabetic patients in 2014. J Educ Community Health 2015; 2: 38-49.

35. Ouji Z, Barati M, Bashirian S. Application of BASNEF model to predict postpartum physical activity in mothers visiting health centers in Kermanshah. J Educ Community Health 2014; 1: 54-62. 